DRAFT VERSION MARCH 27, 2020

Typeset using LATEX twocolumn style in AASTeX63

\title{
Detailed abundances in the Galactic center: Evidence of a metal-rich alpha-enhanced stellar population
}

\author{
B. Thorsbro, ${ }^{1}$ N. Ryde, ${ }^{1}$ R. M. Rich, ${ }^{2}$ M. Schultheis,${ }^{3}$ F. Renaud,${ }^{1}$ E. Spitoni, ${ }^{4}$ T. K. Fritz,${ }^{5,6}$ A. Mastrobuono-Battisti, ${ }^{1}$ \\ L. ORiglia, ${ }^{7}$ F. MATtEUCCI, ${ }^{8,9,10}$ AND R. SCHÖDEL ${ }^{11}$ \\ ${ }^{1}$ Lund Observatory, Department of Astronomy and Theoretical Physics, Lund University, Box 43, SE-22100 Lund, Sweden \\ ${ }^{2}$ Department of Physics and Astronomy, UCLA, 430 Portola Plaza, Box 951547, Los Angeles, CA 90095-1547, USA \\ ${ }^{3}$ Observatoire de la Côte d'Azur, CNRS UMR 7293, BP4229, Laboratoire Lagrange, F-06304 Nice Cedex 4, France \\ ${ }^{4}$ Stellar Astrophysics Centre, Department of Physics and Astronomy, Aarhus University, Ny Munkegade 120, DK-8000 Aarhus C, Denmark \\ ${ }^{5}$ Instituto de Astrofísica de Canarias, Calle Via Láctea s/n, E-38206 La Laguna, Tenerife, Spain \\ ${ }^{6}$ Universidad de La Laguna. Avda. Astrofísico Fco. Sánchez, La Laguna, Tenerife, Spain \\ ${ }^{7}$ INAF-OAS Osservatorio di Astrofisica e Scienza dello Spazio di Bologna, Via Gobetti 93/3,I-40129 Bologna, Italy \\ ${ }^{8}$ Dipartimento di Fisica, Sezione di Astronomia, Università di Trieste, via G.B. Tiepolo 11, I-34131, Trieste, Italy \\ ${ }^{9}$ I.N.A.F. Osservatorio Astronomico di Trieste, via G.B. Tiepolo 11, I-34131, Trieste, Italy \\ ${ }^{10}$ I.N.F.N. Sezione di Trieste, via Valerio 2, 34134 Trieste, Italy \\ ${ }^{11}$ Instituto de Astrofísica de Andalucía (CSIC), Glorieta de la Astronomía s/n, 18008 Granada, Spain
}

\begin{abstract}
We present a detailed study of the composition of $20 \mathrm{M}$ giants in the Galactic center with 15 of them confirmed to be in the Nuclear Star Cluster. As a control sample we have also observed $7 \mathrm{M}$ giants in the Milky Way Disk with similar stellar parameters. All 27 stars are observed using the NIRSPEC spectograph on the KECK II telescope in the $\mathrm{K}$-band at a resolving power of $R=23,000$. We report the first silicon abundance trends versus $[\mathrm{Fe} / \mathrm{H}]$ for stars in the Galactic center. While finding a disk/bulge like trend at subsolar metallicities, we find that $[\mathrm{Si} / \mathrm{Fe}]$ is enhanced at supersolar metallicities. We speculate on possible enrichment scenarios to explain such a trend. However, the sample size is modest and the result needs to be confirmed by additional measurements of silicon and other $\alpha$-elements. We also derive a new distribution of $[\mathrm{Fe} / \mathrm{H}]$ and find the most metal rich stars at $[\mathrm{Fe} / \mathrm{H}]=+0.5$ dex, confirming our earlier conclusions that the Galactic center hosts no stars with extreme chemical composition.
\end{abstract}

Keywords: stars: abundances — nuclear star cluster — silicon — late-type — Galaxy: center

\section{INTRODUCTION}

Nuclear star clusters are ubiquitous in galaxies: They are present in $\gtrsim 70 \%$ of early and late type galaxies with stellar masses above $10^{8}-10^{10} \mathrm{M}_{\odot}$. at greater stellar masses, their frequency decreases in early type galaxies, but stays high in late types. Nuclear star clusters have been found to coexist with supermassive black holes (SMBH), especially at the low-mass range for SMBH. However, there are numerous cases of nuclear star clusters, where a central black hole could not be detected, i.e. has a tight upper mass limit. The masses of nuclear star clusters display scaling relationships with the masses of their host galaxies. This supports the idea that the nuclei of galaxies may have co-evolved with their hosts. Reviews by Neumayer et al. (2020); Böker (2010); Neumayer (2017); Seth et al. (2019) consider nuclear star clusters, their formation history, and their role in the evolution of galaxies.

thorsbro@astro.lu.se
The nuclear star cluster at the center of the Milky Way (NSC) has a half-light radius of about $5 \mathrm{pc}$ (corresponding to about 2 arcmin at the distance of the GC), a mass of $2.5 \pm 0.4 \times 10^{7} \mathrm{M}_{\odot}$, is flattened along the Galactic northsouth direction and rotates in parallel to the Galactic Disc. The Milky Way's central black hole, Sagittarius A* (Sgr A*) is located at the precise center of the NSC. The NSC appears to be very similar to extragalactic NSCs. It is currently the only one where we can observationally resolve individual stars and it therefore plays a key role as a template for its extragalactic counterparts (Fritz et al. 2016; Schödel et al. 2014; Neumayer et al. 2020).

The NSC is not well isolated, but lies embedded into the so-called Nuclear Stellar Disk (NSD), a dense stellar region that has a radius of $100-200 \mathrm{pc}$ and a scale height of about $45 \mathrm{pc}$ (Launhardt et al. 2002). In spite of massive young clusters and other signs of ongoing massive star formation, $80-90 \%$ of the stars in the NSD appear to have formed more than 8 Gyr ago (Nogueras-Lara et al. 2018, 2019a).

Due to the co-penetration and overlap of different stellar components along the ling of sight to the GC (disc, bulge/bar, nuclear disc, nuclear cluster) assigning membership of any 
given star to the NSC is a complex issue. As members of the NSC have orbits that linger at the apocenter, it is not necessary to observe stars very near the black hole to gain insights into the physical properties of the NSC. But contamination of any target sample by stars that are not in the NSC increases as a function of distance from from $\mathrm{Sgr}$ A*.

Two channels have been proposed to account for the presence of NSCs in galaxies (see, e.g. Neumayer 2017; Neumayer et al. 2020). (1) Inspiral via dynamical friction and subsequent merger of globular cluster like systems into the nucleus (Tremaine et al. 1975; Capuzzo-Dolcetta 1993; Antonini et al. 2012). (2) In situ formation of stars, that might result from an extended star formation history, including sharing some history with the formation of the bulge/bar population (Loose et al. 1982; Milosavljević 2004; Mapelli et al. 2012; Mastrobuono-Battisti et al. 2019).(3) Finally, a channel worthy of consideration would be the capture of Galactic bulge stars on ergodic orbits; these stars might plausibly originate in nuclear discs or spirals or in bars.

In the case of the NSC we can test these scenarios by studying its stars. Very young massive stars detected in the central parsec of the Galaxy provides unambiguous evidence for current in situ star formation (Genzel et al. 2010; FeldmeierKrause et al. 2015). Using galaxy simulations, Guillard et al. (2016) proposed a hybrid formation scenario in which clusters bring some gas with them to form a fraction of NSC stars in situ. If drawn from the Milky Way globular cluster system - even that of the bulge - such stars would be expected to contribute stars of predominantly subsolar metallicity. However, young and more metal rich star clusters formed close to the Galactic center may also have contributed to its build up (Arca-Sedda et al. 2015).

The compositions of stars can strongly constrain which of these scenarios is dominant. Early investigations in the Galactic center $\sim$ tens of parsecs distance from Sgr A*, focused on the brightest star in the region, the M2 supergiant IRS 7 (Carr et al. 2000; Davies et al. 2009) and later extended to include other bright targets (Ramírez et al. 2000; Cunha et al. 2007). These studies commondly find $-0.2<$ $[\mathrm{Fe} / \mathrm{H}]<+0.4$ and an $\alpha$-abundance in the range between about 0.0 to 0.5 dex, both in reference to Solar values by Grevesse et al. (2007). Analyzing fainter red giants, most likely located more distant in the NSD, Ryde \& Schultheis (2015); Ryde et al. (2016b) find similar results, but with lower alphas.

Do et al. (2015) used medium resolution, Adaptive Opticsfed spectroscopy to probe the central parsec, and found some metal poor star candidates and an extremely metal rich abundance distribution reaching up to ten times Solar in $[\mathrm{Fe} / \mathrm{H}]$. Metal poor giants were confirmed; the high resolution abundance analysis of a metal poor, alpha-enhanced $\mathrm{M}$ giant 58 pc from SgrA* was conducted by Ryde et al. (2016a). Rich et al. (2017) reported the first high resolution abundance analysis for members of the NSC, finding a wide abundance distribution with mean $[\mathrm{Fe} / \mathrm{H}]=-0.16$ dex extending up to +0.6 dex. Feldmeier-Krause et al. (2017) used medium resolution KMOS spectroscopy to derive metallicities for 700 stars in the central $4 \mathrm{pc}^{2}$. They find a significantly more metal rich distribution (median $0.29 \mathrm{dex}$; extended to $+1 \mathrm{dex}$ ). High resolution spectroscopy of 2 stars in the central parsec resulted in a claim by Do et al. (2018) for extreme abundances of scandium, vanadium, and yttrium in the NSC, along with iron abundances too high to measure with current atmosphere models. While our work does not have access to the central parsec, we did confirm that the lines of scandium, vanadium, and yttrium are strong in cool NSC giants (Thorsbro et al. 2018). However, from our local disk control sample, we find these lines are also strong in cool local disk giants. Thus, the strong lines are a property of cool stars, and we attribute this strength not to an abundance increase but rather to hyperfine splitting (that delays saturation) and temperature sensitive non-LTE strengthening of the line (for more details, see Thorsbro et al. 2018). Najarro et al. (2009) investigated two luminous blue variables in the Quintuplet cluster and find solar metallicity with enhanced $\alpha$-abundances.

Investigations into variable stars are also relevant for the study of the Galactic center as RR Lyrae stars can be tracers of very old stellar populations (Minniti et al. 2016; Dong et al. 2017) and classical Cepheids can be tracers of young stellar populations Matsunaga et al. (2015); Kovtyukh et al. (2019). At present, a handful of RR ab variables are found near the NSC from the Dong et al. (2017) study but their actual membership in the NSC remains a matter of debate.

In general $\alpha$-element abundance trends have not been investigated, which would help constraining different models for the formation and evolution of the inner Galaxy (e.g. Matteucci 2012; Di Matteo 2016). Here we report a new, interesting abundance trend in $[\mathrm{Si} / \mathrm{Fe}]$, from the observations of $20 \mathrm{M}$ giants in the NSC and vicinity and $7 \mathrm{M}$ giants observed in the Milky Way Disk (MWD). This is a continuation of the work started by Ryde et al. (2016a); Rich et al. (2017); Thorsbro et al. (2018).

\section{OBSERVATIONS}

$27 \mathrm{M}$ giants have been observed at medium/high spectral resolution in the K-band using the NIRSPEC (McLean 2005; McLean et al. 2007) facility at Keck II, and are summarized in Table 1 . We used the $0.432^{\prime \prime} \times 12^{\prime \prime}$ slit and the NIRSPEC-7 filter, giving the resolving power of $R \sim 23,000$, which is a minimum for determining accurate abundances from spectra of such cool stars. Five spectral orders are recorded, covering the wavelength range of 21,000-24,000 $\AA$. However, the wavelength coverage is not complete; there are gaps between the orders.

Seven of these observed stars are located in the MWD with similar temperature range to the observed Galactic center stars, thus enabling them to be used as a control sample having similar systematic uncertainties. The MWD stars are presented in Thorsbro et al. (2018), where they are important for the discussion on the strong, K-band Sc I lines detected in Galactic center stars. The other 20 stars are located in the Galactic center, of which 17 are observed under the programs U87NS (April 2015, PI: Rich) and U103NS (April 2016, PI: Rich) are presented in Ryde et al. (2016b); Rich 
Table 1. Compiled data for the observed stars discussed in this paper. We assume solar abundances of $\mathrm{A}(\mathrm{Fe})=7.45$ and $\mathrm{A}(\mathrm{Si})=7.51$ (Grevesse et al. 2007). General uncertainties are listed for each of the stellar parameters and the silicon abundances. The locations are Milky Way Disk (MWD), Nuclear Star Cluster (NSC), and Stellar Disk (NSD).

\begin{tabular}{|c|c|c|c|c|c|c|c|c|c|}
\hline $\begin{array}{c}\text { Star } \\
\text { uncertainties: }\end{array}$ & $\mathrm{K}_{\mathrm{s}}$ & [h:m:s] & [d:m:s] & $\begin{array}{c}T_{\text {eff }} \\
\pm 150 \\
{[\mathrm{~K}]}\end{array}$ & $\begin{array}{l}\log g \\
\pm 0.3\end{array}$ & $\begin{array}{c}{[\mathrm{Fe} / \mathrm{H}]} \\
\pm 0.15 \\
(\mathrm{dex})\end{array}$ & $\begin{array}{c}\xi_{\text {micro }} \\
\pm 0.15 \\
{\left[\mathrm{~km} \mathrm{~s}^{-1}\right]}\end{array}$ & $\begin{array}{l}{[\mathrm{Si} / \mathrm{Fe}]} \\
\pm 0.15 \\
(\mathrm{dex})\end{array}$ & Location \\
\hline 2M17584888-2351011 & 6.49 & $17: 58: 48.89$ & $-23: 51: 01.17$ & 3652 & 1.30 & 0.55 & 2.0 & 0.06 & MWD \\
\hline 2M18103303-1626220 & 6.51 & $18: 10: 33.04$ & $-16: 26: 22.06$ & 3436 & 0.61 & 0.06 & 2.3 & 0.02 & MWD \\
\hline 2M18191551-1726223 & 6.56 & $18: 19: 15.51$ & $-17: 26: 22.35$ & 3596 & 0.84 & -0.02 & 2.2 & 0.00 & MWD \\
\hline $2 \mathrm{M} 18550791+4754062$ & 7.63 & $18: 55: 07.92$ & 47:54:06.22 & 3915 & 1.24 & -0.20 & 2.0 & 0.07 & MWD \\
\hline $2 \mathrm{M} 19122965+2753128$ & 6.60 & $19: 12: 29.66$ & $27: 53: 12.83$ & 3263 & 0.48 & 0.29 & 2.4 & 0.12 & MWD \\
\hline 2M19411424+4601483 & 7.69 & $19: 41: 14.25$ & 46:01:48.14 & 3935 & 1.13 & -0.41 & 2.0 & 0.21 & MWD \\
\hline $2 \mathrm{M} 21533239+5804499$ & 6.58 & $21: 53: 32.40$ & 58:04:49.94 & 3708 & 1.28 & 0.35 & 2.0 & -0.01 & MWD \\
\hline $\mathrm{GC} 25^{a}$ & 10.44 & $17: 45: 40.93$ & $-29: 00: 24.39$ & 3383 & 0.38 & -0.08 & 2.5 & 0.08 & NSC \\
\hline GC 6227 & 11.82 & $17: 45: 38.86$ & $-29: 01: 08.71$ & 3681 & 0.92 & -0.27 & 2.1 & 0.11 & $\mathrm{NSC}^{b}$ \\
\hline GC 7104 & 10.74 & $17: 45: 38.94$ & $-29: 00: 58.44$ & 3657 & 1.09 & 0.30 & 2.0 & 0.20 & NSD \\
\hline GC 8623 & 10.53 & $17: 45: 41.83$ & $-29: 00: 46.15$ & 3433 & 0.38 & -0.12 & 2.5 & 0.07 & $\mathrm{NSC}$ \\
\hline GC 8631 & 11.56 & $17: 45: 43.02$ & $-29: 00: 46.02$ & 3899 & 1.41 & 0.27 & 1.9 & 0.23 & NSC \\
\hline GC 10195 & 10.67 & $17: 45: 43.10$ & $-29: 00: 25.45$ & 3414 & 0.55 & -0.01 & 2.4 & 0.03 & $\mathrm{NSC}^{b}$ \\
\hline GC 10812 & 10.25 & $17: 45: 37.23$ & $-29: 00: 16.62$ & 3895 & 0.60 & -1.02 & 2.3 & 0.41 & NSD \\
\hline GC 11025 & 10.41 & $17: 45: 37.13$ & $-29: 00: 14.39$ & 3359 & 0.64 & 0.25 & 2.3 & 0.25 & NSC \\
\hline GC 11473 & 11.74 & $17: 45: 42.64$ & $-29: 00: 10.23$ & 3520 & 1.06 & 0.38 & 2.0 & 0.26 & NSD \\
\hline GC 11532 & 10.90 & $17: 45: 42.90$ & $-29: 00: 09.60$ & 3450 & 0.48 & -0.23 & 2.4 & 0.09 & $\mathrm{NSC}$ \\
\hline GC 13282 & 10.99 & $17: 45: 39.49$ & $-28: 59: 58.74$ & 3543 & 0.77 & -0.04 & 2.2 & -0.02 & NSC \\
\hline GC 13727 & 10.82 & $17: 45: 39.59$ & $-28: 59: 56.21$ & 3382 & 0.31 & -0.28 & 2.5 & 0.15 & NSC \\
\hline GC 13882 & 10.56 & $17: 45: 38.70$ & $-28: 59: 55.15$ & 3344 & 0.35 & -0.17 & 2.5 & 0.01 & $\mathrm{NSC}$ \\
\hline GC 14024 & 10.99 & $17: 45: 42.03$ & $-28: 59: 54.97$ & 3450 & 0.96 & 0.49 & 2.1 & 0.15 & NSC \\
\hline GC 14724 & 10.56 & $17: 45: 37.31$ & $-28: 59: 49.43$ & 3482 & 0.45 & -0.32 & 2.4 & 0.14 & NSC \\
\hline GC 15540 & 10.49 & $17: 45: 41.93$ & $-28: 59: 23.39$ & 3496 & 0.61 & 0.01 & 2.3 & 0.04 & NSC \\
\hline GC 16763 & 10.66 & $17: 45: 37.29$ & $-29: 01: 12.67$ & 3510 & 0.58 & -0.15 & 2.3 & -0.03 & NSC \\
\hline GC 16867 & 11.77 & $17: 45: 36.02$ & $-29: 00: 09.20$ & 3628 & 0.76 & -0.18 & 2.2 & 0.02 & $\mathrm{NSC}$ \\
\hline GC 16887 & 11.63 & $17: 45: 44.04$ & $-28: 59: 27.66$ & 3308 & 0.77 & 0.41 & 2.2 & 0.18 & NSC \\
\hline GC 16895 & 10.75 & $17: 45: 35.64$ & $-29: 00: 47.00$ & 3438 & 0.50 & -0.13 & 2.4 & 0.07 & NSC \\
\hline
\end{tabular}

${ }^{a}$ GC 25 appears in other works and is listed as $I d=44$ in Feldmeier-Krause et al. (2017) and Name=NE1-1 001 in Do et al. (2015); Støstad et al. (2015). Note that the star listed as GC 25 in Nandakumar et al. (2018) is not the same star in spite of having the same name.

${ }^{b}$ GC 6227 and GC 10195 are possible background stars depending on whether they are intrinsically reddened. 
et al. (2017). Figure 1 shows a JHKs RGB image from the GALACTICNUCLEUS survey (Nogueras-Lara et al. 2019b) of the observed stars. Figure 2 shows a color-magnitude diagram (CMD) with our Galactic center $\mathrm{M}$ giants, color-coded with the derived metallicities (see Sect. 3.4), superimposed on the 2MASS sample of the Galactic Center field. Our target stars have been selected over the full width of the CMD in order to avoid any selection bias. Note that the star listed as GC 7688 in Rich et al. (2017) is not included as it has been identified as a foreground bulge star. Three stars have been added to the sample, all of them observed on 2017 July 28-29, under program U103NS (PI: Rich). These are selected in the same way as the 17 original stars from low resolution SINFONI spectra (see Rich et al. 2017). Similarly, these are observed with an ABBA scheme with a nod throw of $6^{\prime \prime}$ along the slit, in order to achieve proper background and dark subtraction. The three added stars have each been exposed for 2000 s. These stars are also reduced with the NIRSPEC software redspec (Kim et al. 2015), and thereafter with IRAF (Tody 1993) to normalize the continuum, eliminate cosmic ray hits, and correct for telluric lines. The latter has been done with a high signal-to-noise spectrum of the rapidly rotating O6.5V star HIP89584. For more details about the data reduction process, we refer to Rich et al. (2017).

\section{ANALYSIS}

Our goal is to determine the $[\alpha / \mathrm{Fe}]$-ratios as a function of metallicity for stars in the NSC and its vicinity. However, the spectra of the Galactic center stars show a surprising amount of features due to the presence of diatomic molecules, in particular the $\mathrm{CN}$ molecule. To perform a good abundance analysis it is important to make sure all spectral features are identified so a line list containing unblended lines can be constructed for good abundance analysis, see Section 3.1.

We analyze the $M$ giant spectra using tailored synthetic spectra, calculating the radiative transfer through spherical model atmospheres. In order to derive as accurate abundances as possible, the fundamental input parameters for these stellar atmospheres are needed to be well determined. These parameters are the effective temperature, $T_{\text {eff }}$, surface gravity, $\log g$, metallicity, $[\mathrm{Fe} / \mathrm{H}]$, and the microturbulence, $\xi_{\text {micro }}$, of the stars. These are discussed in Sections 3.2-3.4.

In an earlier study, we analyzed a giant with $[\mathrm{Fe} / \mathrm{H}] \sim-1$ found in the vicinity of the NSC (Ryde et al. 2016b), measuring abundances for $\mathrm{Mg}, \mathrm{Si}, \mathrm{Ca}$, and $\mathrm{Ti}$. The remaining NSC giants are cooler and more metal rich; molecules and blending severely limits the usable lines so that at our resolution so we are able only to measure the $\alpha$-element $\mathrm{Si}$ (see Section 3.5).

From three KECK runs, during the period 2015-2017, we have gathered spectra of a sample of $20 \mathrm{M}$ giants that are all shown to be confined to the NSC and near vicinity (see Section 3.6).

\subsection{Line List}

In order to synthesize the spectra, an accurate line list is required. In the list provided by Thorsbro et al. (2017) wave- lengths and line-strengths (astrophysical $\log g f$-values) are updated using the solar center intensity atlas (Livingston \& Wallace 1991). We have also made use of recent laboratory measurements of atomic line strengths of $\mathrm{Mg} \mathrm{I}$, and $\mathrm{Sc} \mathrm{I}$ (Pehlivan Rhodin et al. 2017; Pehlivan et al. 2015), as well as of Si I (Pehlivan Rhodin et al., 2020, submitted) in the K band. Of approximately 700 identified, interesting spectral lines for cool stars, about 570 lines have been assigned new values.

As molecular lines have a crucial impact in terms of blending, we include molecular lines in the line list. For $\mathrm{CN}$ we include the list from Sneden et al. (2016), which is the most dominant molecule apart from $\mathrm{CO}$, whose lines dominate in the $2.3 \mu \mathrm{m}$ bandhead region. The $\mathrm{CO}$ line data are from Goorvitch (1994). At the shorter wavelengths of our spectra region $\mathrm{SiO}, \mathrm{H}_{2} \mathrm{O}$, and $\mathrm{OH}$ are important. Line lists for these molecules are included into our line list from respectively Langhoff \& Bauschlicher (1993); Barber et al. (2006); Brooke et al. (2016).

To identify clean spectral lines suitable for measurement, we examine synthetic model spectra of cool $\mathrm{M}$ giants to identify iron lines that are not blended with molecular features. The CN line list used (Sneden et al. 2016) has been shown to reproduce the observed $\mathrm{CN}$ spectrum very well, and can be used to identify blends. Unknown blends might still always be present and special care has been taken when such possible mismatches are identified. Examples of lines that are not blended are illustrated in Figure 3, where an Fe I line and a Si I line are shown.

For the determination of $\mathrm{Fe}$ and $\mathrm{Si}$ abundances the lines shown in Table 2 have been identified as good lines with little or no blending.

\subsection{Effective Temperature, $T_{\mathrm{eff}}$}

The effective temperatures of the stars in the MWD are obtained following Thorsbro et al. (2018), where the temperatures from the APOGEE Data Release 14 (Majewski et al. 2017; Abolfathi et al. 2018; Blanton et al. 2017) were examined and then adopted.

To determine the temperatures of the stars in the Galactic center we rely on the method of Schultheis et al. (2016) as employed in Rich et al. (2017), which is based on using low-resolution SINFONI (Eisenhauer et al. 2003) spectra and measures an index of the $\mathrm{CO}$ band head at $2.3 \mu \mathrm{m}$. Rich et al. (2017) determined the effective temperatures for 17 of the 20 Galactic center stars in our sample. The same method is used for determining the temperature for the added stars, namely GC 8623 and GC 13727. We do not have SINFONI spectra for the GC 25 star and thus it remains undetermined using the $\mathrm{CO}$ band head method. The $\mathrm{CO}$ band head temperatures are shown in Table 3 in the "CO band" column.

To decrease the uncertainty in the determined temperatures for the stars in the Galactic center and to increase the correlation between the temperatures of the stars in the MWD stars and the stars in the Galactic center, we use the fact that there are strong Sc I lines in the K-band spectrum that are very temperature sensitive in the $3000-4000 \mathrm{~K}$ range (e.g. Thors- 


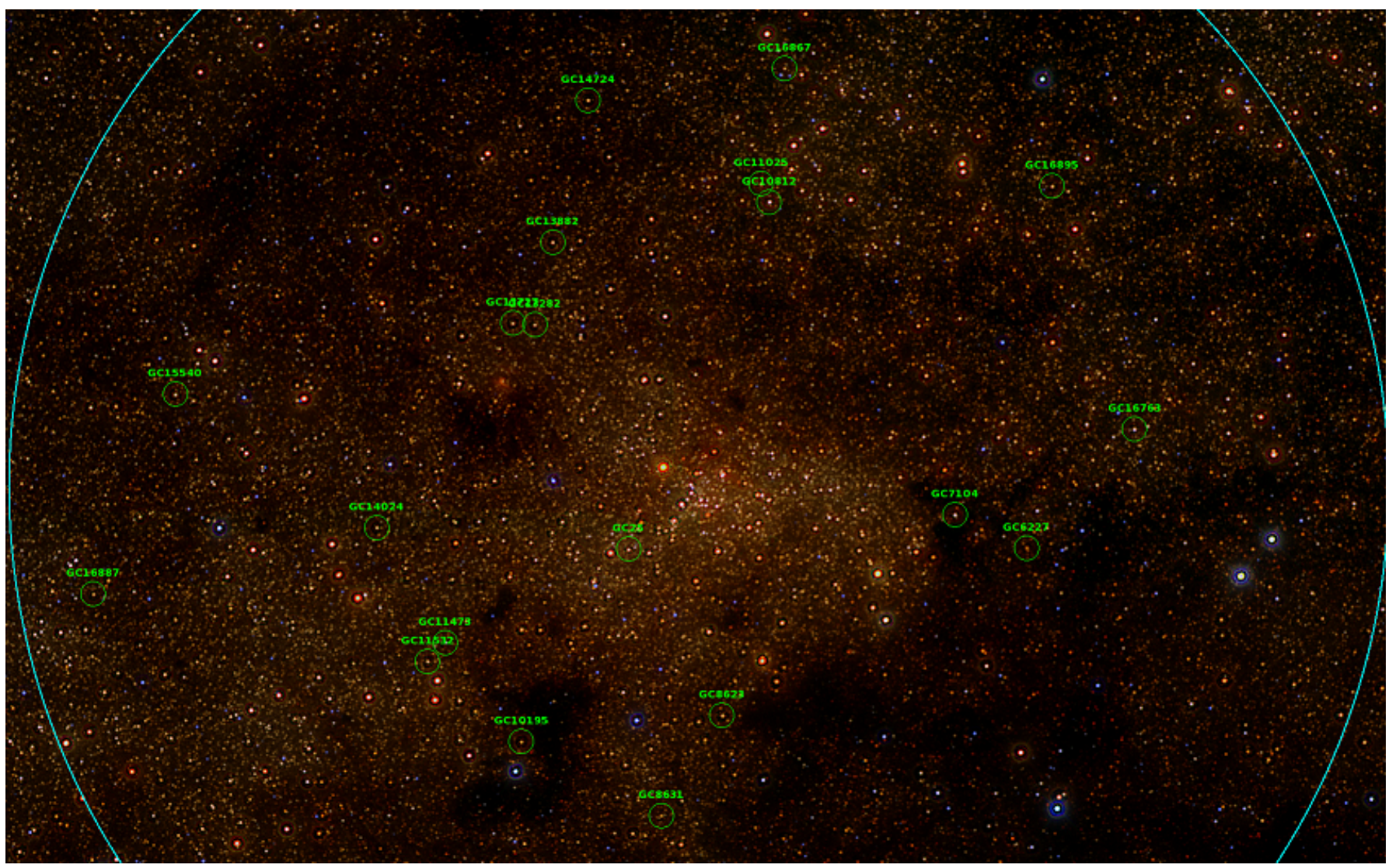

Figure 1. A JHKs RGB image from the GALACTICNUCLEUS survey (Nogueras-Lara et al. 2018, 2019b) marking the observed stars in the region inside $3.5 \mathrm{pc}$ projected distance from $\mathrm{Sgr}$ A* illustrated with the blue circle.

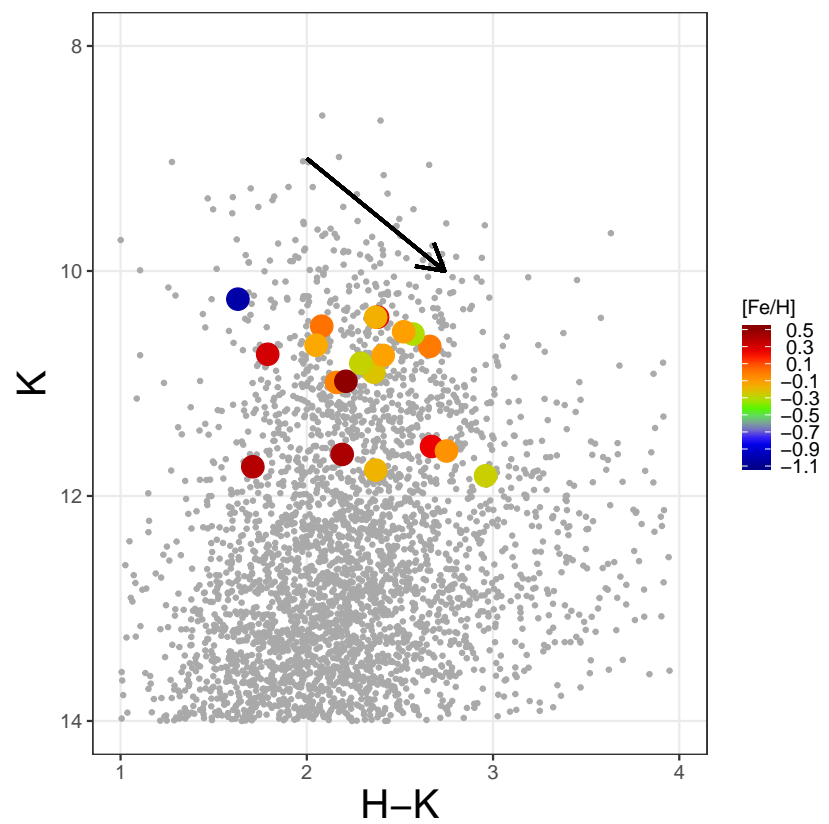

Figure 2. 2MASS K vs H-K diagram in the NSC superimposed by our sample of $20 \mathrm{M}$ giants colored by metallicity (see Sect. 3.4).

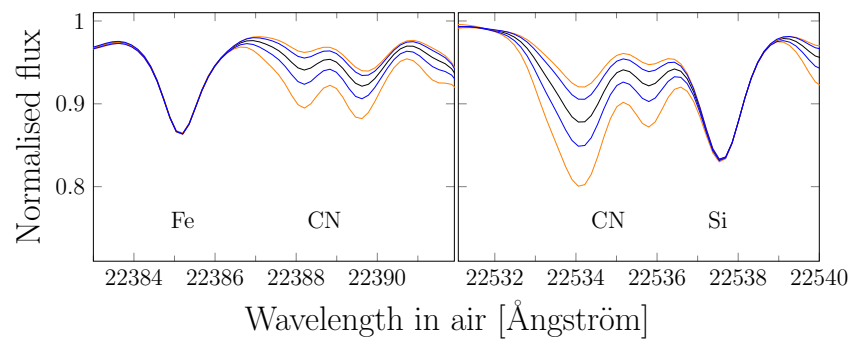

Figure 3. Synthetic model spectra for a star with solar metallicity at $3600 \mathrm{~K}$ effective temperature. The black line shows typical $\mathrm{M}$ giant abundances of carbon and nitrogen, the blue lines show the effect of varying the nitrogen abundance by $\pm 0.2 \mathrm{dex}$, and the orange lines show a variation in the carbon abundance of $\pm 0.2 \mathrm{dex}$. The Fe I line shown in the left plot and the Si I line in the right plot are not blended with $\mathrm{CN}$ molecules. For the stars we are investigating, $\mathrm{CN}$ blends are very common in the near infrared, so finding unblended lines is crucial.

bro et al. 2018). The effects of different scandium abundances, surface gravities or metallicities are a second order effect. Most of our observed stars are within this temperature range and the strengths of these lines should therefore be able to be used as a temperature indicator. We measure the equivalent widths for a set of scandium lines in the MWD stars 
Table 2. Lines used for abundance determinations.

\begin{tabular}{lrr}
\hline \hline $\begin{array}{r}\text { Wavelength in air } \\
{[\AA]}\end{array}$ & $\begin{array}{c}E_{\text {exc }} \\
{[\mathrm{eV}]}\end{array}$ & \multicolumn{1}{c}{$\log g f$} \\
\hline Fe I & & \\
21178.211 & 3.0173 & -4.201 \\
21238.509 & 4.9557 & -1.281 \\
21284.348 & 3.0714 & -4.414 \\
21851.422 & 3.6417 & -3.506 \\
22380.835 & 5.0331 & -0.409 \\
22385.143 & 5.3205 & -1.536 \\
22392.915 & 5.0996 & -1.207 \\
22419.976 & 6.2182 & -0.226 \\
\hline Si I & & \\
21354.249 & 6.2228 & 0.138 \\
21368.693 & 7.3288 & -1.905 \\
21874.199 & 6.7206 & -0.731 \\
21879.345 & 6.7206 & 0.384 \\
21924.981 & 7.0645 & -1.269 \\
22537.593 & 6.6161 & -0.216 \\
\hline
\end{tabular}

for which the effective temperatures are known. We create four linear relations between the effective temperature and the equivalent widths of the four scandium lines at the following wavelengths in air: $21730 \AA, 21812 \AA, 21842 \AA$, and $22394 \AA$. Using the equivalent widths measured of the same scandium lines in the spectra of the Galactic center stars together with these empirical relations, and taking the average of the four results (having standard deviations on the order of $50 \mathrm{~K}$ ), we can thus determine the effective temperature of the Galactic center stars. The effective temperatures determined this way are given in Table 3 under the column "Scandium".

Comparing the effective temperature values found in Table 3 , and with a general uncertainty of 150 to $200 \mathrm{~K}$, we find that the results from the different methods are in general agreement. We decide to take an average of the values, and keep a conservative estimate of $150 \mathrm{~K}$ uncertainty for the effective temperature values. These temperatures are given in the "Mean" column in Table 3. For GC 25, we use the temperature determined from the $\mathrm{Sc} I$ lines.

\subsection{Surface Gravity, $\log g$, and Microturbulence, $\xi_{\text {micro }}$}

Rich et al. (2017) demonstrated that in spite of the distance to the stars in the Galactic center is known to a good degree, the photometrically determined surface gravities are plagued with large uncertainties due to the patchy extinction. To determine surface gravity, $\log g$, we instead benefit from the fact that isochrones of old ( $>1 \mathrm{Gyr}) \mathrm{M}$ giants are quite insensitive to the age of the star. This reduces isochrones to depend
Table 3. Analysis of the effective temperature of stars in the Galactic center.

\begin{tabular}{|c|c|c|c|}
\hline Star & $\begin{array}{c}\text { CO band } \\
T_{\text {eff }} \\
{[\mathrm{K}]}\end{array}$ & $\begin{array}{c}\text { Scandium } \\
\qquad \begin{array}{c}T_{\text {eff }} \\
{[\mathrm{K}]}\end{array}\end{array}$ & $\begin{array}{c}\text { Mean } \\
T_{\text {eff }} \\
{[\mathrm{K}]}\end{array}$ \\
\hline GC 25 & $\ldots$ & 3383 & 3383 \\
\hline GC 6227 & 3800 & 3561 & 3681 \\
\hline GC 7104 & 3750 & 3563 & 3657 \\
\hline GC 8623 & 3415 & 3451 & 3433 \\
\hline GC 8631 & 3900 & 3897 & 3899 \\
\hline GC 10195 & 3500 & 3327 & 3414 \\
\hline GC 10812 & 3800 & 3990 & 3895 \\
\hline GC 11025 & 3400 & 3318 & 3359 \\
\hline GC 11473 & 3550 & 3489 & 3520 \\
\hline GC 11532 & 3500 & 3399 & 3450 \\
\hline GC 13282 & 3700 & 3386 & 3543 \\
\hline GC 13727 & 3372 & 3391 & 3382 \\
\hline GC 13882 & 3300 & 3387 & 3344 \\
\hline GC 14024 & 3650 & 3249 & 3450 \\
\hline GC 14724 & 3600 & 3364 & 3482 \\
\hline GC 15540 & 3600 & 3391 & 3496 \\
\hline GC 16763 & 3600 & 3419 & 3510 \\
\hline GC 16867 & 3650 & 3606 & 3628 \\
\hline GC 16887 & 3500 & 3116 & 3308 \\
\hline GC 16895 & 3450 & 3425 & 3438 \\
\hline
\end{tabular}

on surface gravity, effective temperature, and metallicity. We thus use a grid of Yonsei-Yale isochrones (Demarque et al. 2004) to determine $\log g$ given an effective temperature and metallicity. The construction and validation of this method is described in more detail in Rich et al. (2017).

The microturbulence, $\xi_{\text {micro, }}$, that takes into account the small scale, non-thermal motions in the stellar atmospheres, is important for saturated lines, influencing their line strengths. We estimate this parameter from an empirical relation with the surface gravity based on a detailed analysis of spectra of five red giant stars $(0.5<\log g<2.5)$ by Smith et al. (2013), as described in Rich et al. (2017).

The estimated uncertainty of $150 \mathrm{~K}$ for the effective temperatures propagates into an uncertainty of 0.3 for $\log g$ and $0.15 \mathrm{~km} \mathrm{~s}^{-1}$ for $\xi_{\text {micro }}$.

\subsection{Metallicity, $[\mathrm{Fe} / \mathrm{H}]$}

The metallicities of our stars are important not only for identifying the model atmosphere for a certain star but also for the Metallicity Distribution Function (MDF), which is an 


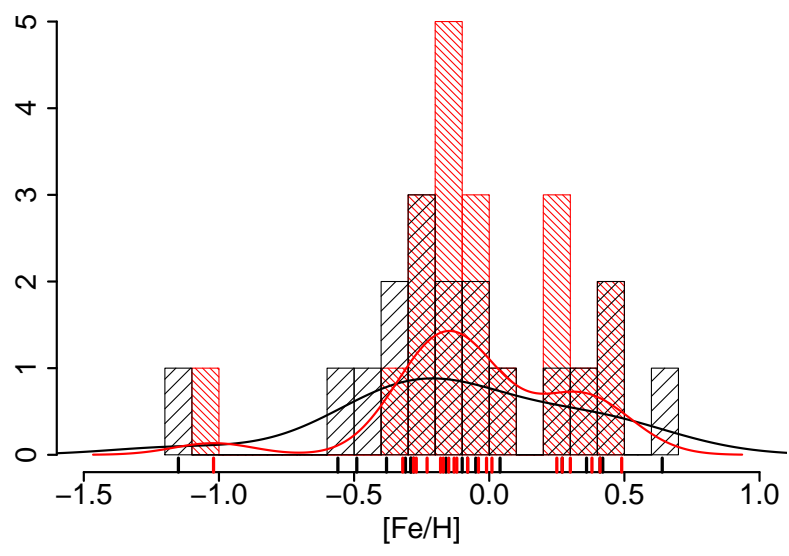

Figure 4. Determined metallicity distribution function (MDF) in red compared to that of Rich et al. (2017) in black. The reanalyzes of the stellar parameters in this work does not significantly change the MDF of the Nuclear Stellar Cluster.

important diagnostic for the discussion of the origin of the Nuclear Star Cluster, see Sect. 4.

In order to determine metallicities we synthesize spectra and compare them to the observed spectra. We have chosen to use the spectral synthesis code Spectroscopy Made Easy (SME) (Valenti \& Piskunov 1996, 2012) which interpolates in a grid of one-dimensional (1D) MARCS atmosphere models (Gustafsson et al. 2008). These are hydrostatic model atmospheres in spherical geometry, computed assuming LTE, chemical equilibrium, homogeneity, and conservation of the total flux (radiative plus convective, the convective flux being computed using a mixing-length recipe). This code has the advantages that it includes a flexible $\chi^{2}$ minimization tool for finding the solution that fits an observed spectrum the best in a pre-specified spectral window. It also has a powerful continuum normalization routine. In cool star spectra, extra care is needed to normalize the spectrum in the region of a spectral line under consideration. The ubiquitous molecules and unavoidable residuals from the telluric line division necessitates a careful analysis line by line.

We recognize that using 3D atmospheric models would be an improvement over 1D atmospheric models (Scott et al. $2015 b, a)$. However, for this differential study we expect to see the same systematics to be present in both the Galactic center and the MWD stellar samples.

As a result of our updated temperatures and careful work on the line list, especially the new analysis of lines blended with $\mathrm{CN}$, we reanalyze the $[\mathrm{Fe} / \mathrm{H}]$ values given in Rich et al. (2017). The new $[\mathrm{Fe} / \mathrm{H}]$ values are shown in Table 1, with a general estimated uncertainty of 0.15 dex. A comparison of our new $[\mathrm{Fe} / \mathrm{H}]$ distribution function $(\mathrm{MDF})$ with that of Rich et al. (2017) is shown in Figure 4.

Principally due to our new treatment of $\mathrm{CN}$ blending, our iron abundances have changed compared to earlier work; for example, the most metal rich star now is $[\mathrm{Fe} / \mathrm{H}]=+0.5$, com-

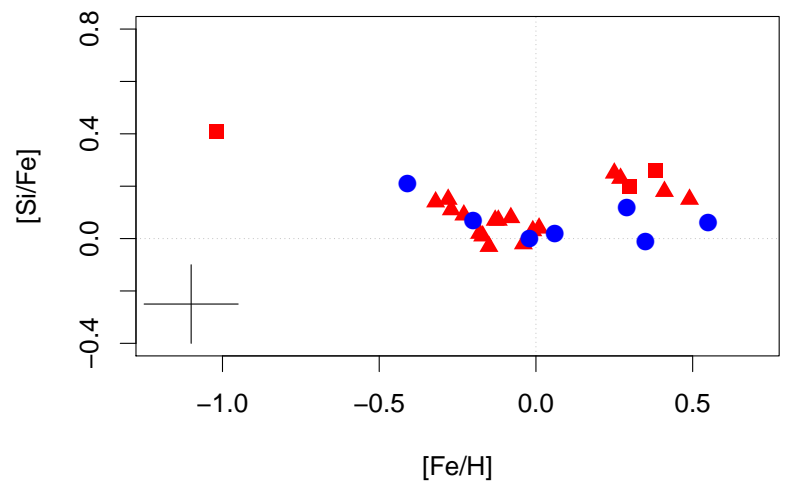

Figure 5. $[\mathrm{Si} / \mathrm{Fe}]$ vs. $[\mathrm{Fe} / \mathrm{H}]$. Red triangles and squares are $\mathrm{Nu}-$ clear Star Cluster respective Nuclear Stellar Disk stars, blue disks the Milky Way Disk stars.

pared to +0.64 in Rich et al. (2017). We consider the present abundance distribution reported in this work to be the best current measurement of $[\mathrm{Fe} / \mathrm{H}]$ and its range in the NSC.

\section{5. $\alpha$-element Abundance Determination}

The $\alpha$-element abundances can potentially be measured from several lines from different species. However, considering the line strengths and number of unblended lines, there are only a few elements possible in the available wavelength region at the spectral resolution considered. The available $\mathrm{Mg}$ I lines are heavily blended with $\mathrm{CN}$ molecular lines and the strengths of the Ti I and Ca I lines increase conspicuously with cooler effective temperature, broadly similar to the Sc I lines discussed in detail by Thorsbro et al. (2018). This behavior argues strongly for non-LTE effects affecting the line strengths more than the abundances. Since no non-LTE corrections are available for these elements in the temperature range that our stars span, these lines cannot yet be used for determining the abundances. Si I, however, has several suitable lines and most importantly, non-LTE departure coefficient calculations show that departure from LTE is not important for $\mathrm{Si}$ I lines in the stellar parameter space of the stars in our sample. We verified this by examining the departure coefficients in MARCS model giants stars, using the atomic model presented in Amarsi \& Asplund (2017), which utilizes the rather efficient inelastic hydrogen collisions of (Belyaev et al. 2014).

The $\alpha$-element abundance is therefore determined from the unblended Si I lines available (Table 2) with a general estimated uncertainty of 0.15 dex. Typical spectra are shown in Figure 6. The silicon abundances are shown in Figure 5 with the Galactic center stars marked with red triangles and squares, the latter for those not identified to be in the NSC (see Section 3.6) and the MWD stars with blue disks.

We now examine more closely those metal rich stars with Si enhancement. Figure 6 shows our observed spectra for the 


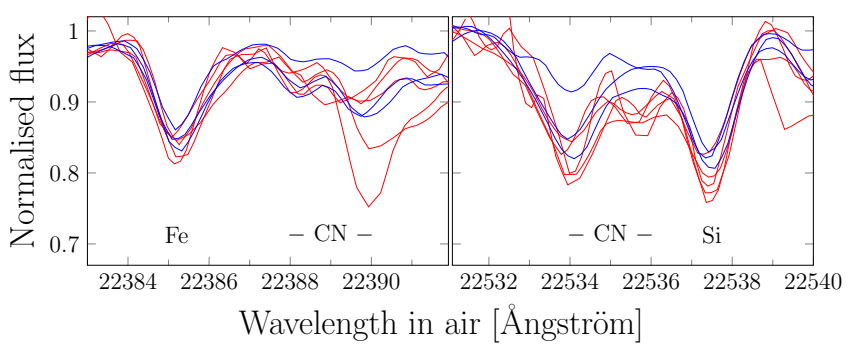

Figure 6. Observed spectra of the subset of stars from our sample with super solar metallicities showing an unblended Fe I line and an unblended Si I line. Blue spectra from Milky Way Disk stars, and red spectra for Galactic center stars. The Si I lines are visibly deeper for the Galactic center stars compared to the Milky Way Disk stars, while the Fe I lines show negligible variation.

metal rich subset giving an extra sanity check on the abundance results. The plot indicates stronger silicon features when comparing the Galactic center stars to the MWD stars, but the Fe I line does not show the strong difference we see in the Si I line. This figure shows that, independent of any abundance analysis, we can see that Si appears to be enhanced in the Galactic center for this subset of stars.

\subsection{Stellar dynamics and positions}

We examine the stellar dynamics of the three stars added to our data set to verify if they belong to the NSC. Secondly, we also check with "metal rich" stars, $[\mathrm{Fe} / \mathrm{H}]>0.1$, differ in their position or dynamics.

As in Ryde et al. (2016a) and Rich et al. (2017) we use the $\mathrm{H}-\mathrm{K}$ color which is nearly only caused by extinction for testing whether the stars are currently in the NSC in the lineof-sight direction. We find that the $v_{\text {rad }}^{\text {LSR }}$ for the added stars GC 25, GC 8623 and GC 13727 are respectively 83, -20 and $-125 \mathrm{~km} \mathrm{~s}^{-1}$ from the Keck spectra. These velocities are consistent with the lower precision values in Fritz et al. (2016). For the proper motions we use Fritz et al. (2016) as previously. The new stars are all likely members of the NSC, see Figure 7.

As visible in Figure 7 we do not find differences between the three added stars or the different metallicity groups. All stars seem to belong to the same hot system with a weak rotation aligned with the Galaxy. The sample, especially of stars with proper motions, is still too small to detect minor differences. We note that three stars, GC 10812, GC 7104 and GC 11473 , have a preferred location in the NSD, of which the latter two are in the metal rich set. Two of the stars GC, 6227 and GC 10195, could possible be background stars, depending of if they are intrinsically reddened. While effects like motion can lead to exchange between the NSC and the NSD, it is still prudent to account for the possibility that some of these stars may reside in the NSD when explaining the enrichment pattern. However, there is still a need for a Galaxy model which includes all four central components (Sgr A*, the NSC, the NSD, and the bar/bulge) to better constrain the location of the stars. Mixing of stars is also a concern to take
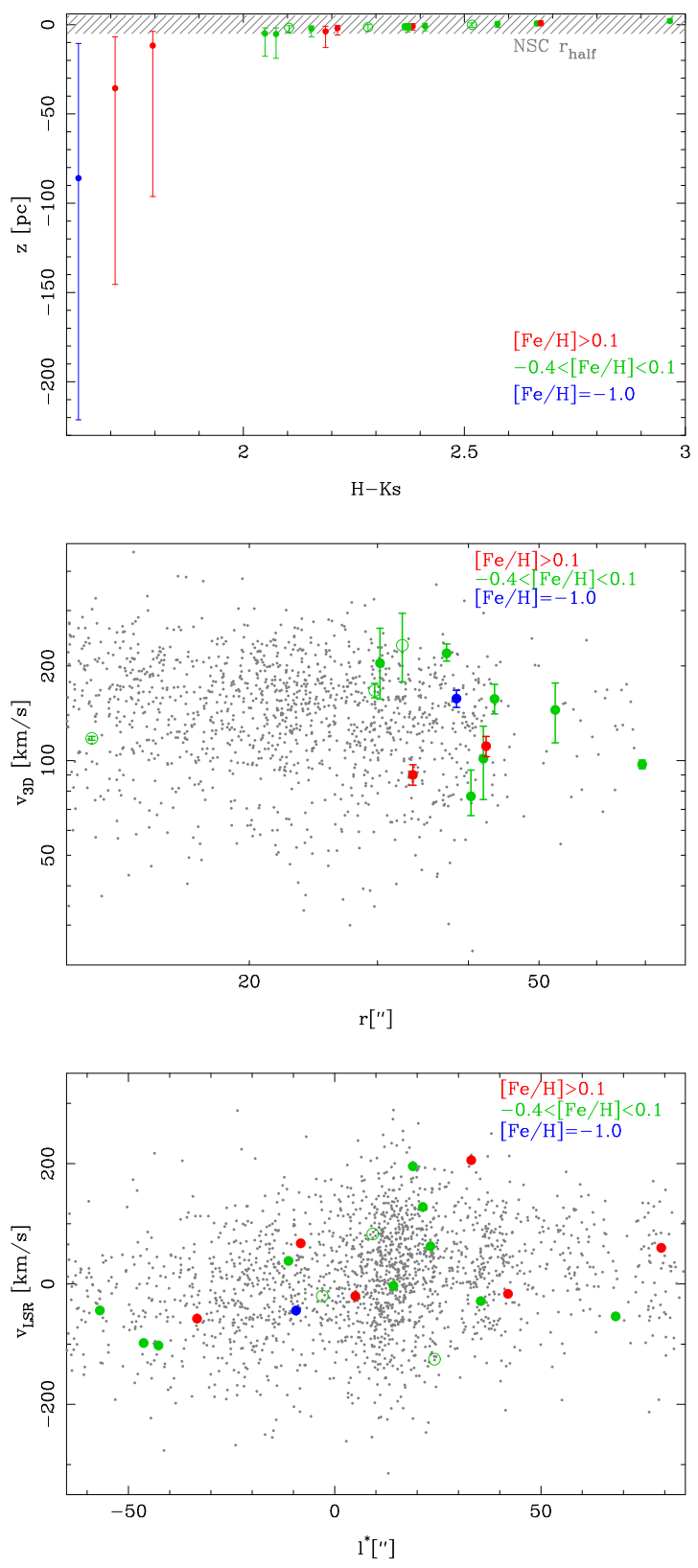

Figure 7. Comparison of sample stars in dynamic and position properties, in all plots the three new stars are plotted as triangles and the old as solid circle. The color indicates the metallicity group. Top: The line-of-sight position of all stars compared to the NSC. The NSD covers the full displayed range. Middle: Total velocity; not all stars are shown here, because some are missing proper motions. Bottom: LSR corrected line-of-sight velocity versus Galactic longitude relative to Sagr A*. Only stars outside the central 10 arcseconds are shown, since there the black hole increases velocities. 
note of, at the projected distance of $2 \mathrm{pc}$ from $\mathrm{Sgr}$ A* there may be about 1 NSD star for every 3-4 NSC stars (GallegoCano et al. 2020).

In the line of sight it seems that there could be a larger metallicity range in the NSD than in the NSC, since of the three stars there, none is a normal metallicity stars. Given the low number of stars, it could be chance also, since Do et al. (2015) and Feldmeier-Krause et al. (2017) find metal poor stars which are at least projected NSC members.

\section{RESULTS AND DISCUSSION}

A new analysis of iron lines including the most comprehensive currently available $\mathrm{CN}$ line list confirms that our sample's most metal rich star has $[\mathrm{Fe} / \mathrm{H}] \sim+0.5$. Our work continues the trend of recent work (Ryde \& Schultheis 2015; Rich et al. 2017; Nandakumar et al. 2018) that finds stars with metallicity higher than Solar, but finds no evidence in the NSC for extreme metallicities. We therefore can not confirm the assertion of Do et al. (2015); Feldmeier-Krause et al. (2017) that stars in the NSC have $[\mathrm{Fe} / \mathrm{H}]$ at or very close to +1 dex.

We have also reported the first trend of $[\mathrm{Si} / \mathrm{Fe}]$ vs. $[\mathrm{Fe} / \mathrm{H}]$ for giants in the NSC and its vicinity. Below Solar metallicity, we largely confirm the initial study of similar giants in the nuclear disk by Ryde \& Schultheis (2015). However, our new results show for the first time a jump in $[\mathrm{Si} / \mathrm{Fe}]$ to $+0.2 \mathrm{dex}$, for $[\mathrm{Fe} / \mathrm{H}]>0$. This particular trend has not been observed in any other stellar population. We emphasize that the sample size is small and the uncertainties of the abundances for $\mathrm{Si}$ and $\mathrm{Fe}$ in quadrature is $\sim 0.2 \mathrm{dex}$, so it is clear that a confirmation using a larger sample, and additional $\alpha$-element lines is an essential next step. In Figure 8 we compare the $[\mathrm{Si} / \mathrm{Fe}]$ abundance ratio as a function of $[\mathrm{Fe} / \mathrm{H}]$ distribution of our samples of NSC (red triangles), NSD (red squares), and MWD (blue triangles) stars with those of giants (gray dots) (Johnson et al. 2014) and microlensed dwarfs (black dots) in the bulge (Bensby et al. 2013). We find that that our NSC and MWD samples fit the lower envelope of the bulge star distribution up to about solar $[\mathrm{Fe} / \mathrm{H}]$. The supersolar stars in the NSC and NSD show enhanced Si abundance compared to our disk and bulge stars.

It is also interesting to compare the properties of the NSC with those of the complex stellar system Terzan 5 in the bulge, which has multiple populations spanning $1.3 \mathrm{dex}$ in metallicity (e.g. Origlia et al. 2011, 2013). Of special note are the two populations at $[\mathrm{Fe} / \mathrm{H}]=-0.3 \mathrm{dex}$ and $[\mathrm{Fe} / \mathrm{H}]=+0.3 \mathrm{dex}$; these two populations have $[\mathrm{Si} / \mathrm{Fe}]=+0.34$ and $[\mathrm{Si} / \mathrm{Fe}]=+0.03$ respectively, with some stars in the metal rich group have subsolar $[\mathrm{Si} / \mathrm{Fe}]$. The ages of these populations have been constrained by Ferraro et al. (2016) and Origlia et al. (2019). The subsolar component with $\alpha$ enhancement was dated at $12 \mathrm{Gyr}$, while a younger turnoff of age 4.5 Gyr appears to be responsible for the metal rich component with about solar-scaled alpha. The trend in $[\alpha / \mathrm{Fe}]$ vs. $[\mathrm{Fe} / \mathrm{H}]$ appears reversed in the NSC compared to Terzan 5, with the $\alpha$-enhancement rising at high metallicity, but other $\alpha$-elements need to be measured before drawing firm conclu-

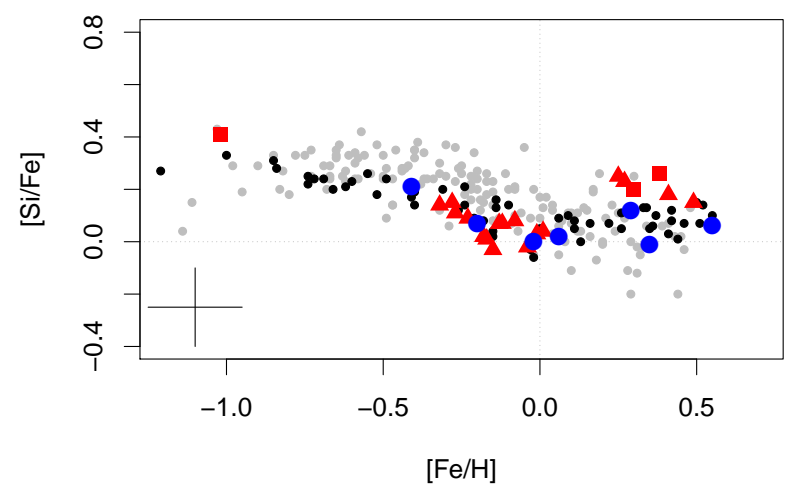

Figure 8. $[\mathrm{Si} / \mathrm{Fe}]$ vs. $[\mathrm{Fe} / \mathrm{H}]$. Red triangles and squares are $\mathrm{Nu}-$ clear Star Cluster respective Nuclear Stellar Disk stars, blue disks the Milky Way Disk stars, gray dots bulge giants from Johnson et al. (2014), black dots microlensed bulge dwarfs from Bensby et al. (2013).

sions. The contrast of these two systems is of great interest, and illustrates how chemical evolution can play out differently, even in systems of very similar metallicity.

\subsection{Chemical Enrichment Scenarios}

Assuming that the enhancement in $\mathrm{Si}$ is also present in other $\alpha$-elements, the origin of the NSC populations is even more intriguing and we can speculate on possible formation and chemical enrichment scenarios. Some of the key questions for this investigation is ages of stars and the source of gas for star formation.

As per the discussion on Terzan 5 above, the subsolar and supersolar metallicities stars of the NSC can be seen as two groups of stars, and it would be interesting to ponder if there is a clear age dichotomy between them. Unfortunately, isochrone dating of the NSC is not possible because currently we can only observe bright giants near the tip.

We use chemical evolution models (Matteucci 2012; Grieco et al. 2015) to explore the chemical enrichment history of the NSC as traced by the sub-solar metallicity stars with about solar-scaled alphas and super-solar metallicity stars with enhanced $\alpha$-elements. The models assume that at the very early epoch of the galaxy and bulge formation a star formation episode occurred with a punctual (and probably local) high star formation rates, i.e. a mini-starburst activity. The type-II supernovae formed during such a burst would then inject $\alpha$-elements within a few Myr. The depth of the gravitational potential well of the Galactic center could help retain or rapidly re-accrete this enriched gas (Emsellem et al. 2015), and possibly form the population presented above, before the outbreak of type-Ia supernovae (that would lower the $[\alpha / \mathrm{Fe}]$ content of the interstellar medium). This early and massive star forming episode could be followed by a recent starburst occurred a few Gyr ago (Pfuhl et al. 2011; Nogueras-Lara et al. 2019a, Schödel et al. 2020 in prep.), 
but the origin of the gas and its retention in the NSC remain a challenge.

We first explore a model where a starburst is initiated by a sudden accretion of gas, either primordial or slightly enriched, which results in a dilution of chemical abundances (e.g. Fe). The model is illustrated in Figure 9, where the dilution of chemical abundances appears on the plot as a horizontal line followed by an increase of the $[\alpha / \mathrm{Fe}]$ ratio. This is due to the contribution of core-collapse SNe II in the starburst, followed by a decrease due to the Fe production from Type Ia SNe. This behavior appears as a loop, as one can see in Figure 9. This kind of behavior has been shown also by Johnson \& Weinberg (2019) studying starbursts and by Spitoni et al. (2019) and Calura \& Menci (2009) in connection with the Galactic disk. In this scenario the super-solar NSC stars could be either old or as young as the sub-solar stars.

To trigger a recent starburst episode, gas needs to be accreted either from other regions of the Galaxy or from an extragalactic origin. In the former case, torques from the bar drive gas inflows toward the center. It is however not clear why such inflow should be intermittent (as required to explain a sudden burst). Simulations of the Milky Way show that discrete accretion events could be due to the fragmentation of the inflowing gas from the Central Molecular Zone (CMZ) at the inner Lindblad resonance (Renaud et al. 2013; Krumholz \& Kruijssen 2015). The other possibility is the accretion of gas from extragalactic origin, for instance from a satellite galaxy tidally stripped during a passage near the Milky Way's center, i.e. on a rather radial trajectory. The gas stripped from a low-mass galaxy would have very low metallicity, whereas the gas from the CMZ could be near or above solar metallicity (Morris \& Serabyn 1996).

Alternatively, we explore a different chemical evolution model where we suppose a pause in the star formation (see Figure 10 where pauses of several lengths are assumed). In these cases, no extra infall event is assumed, but simply that the star formation starts again after the stop. As one can see from Figure 10, in this case there is also a loop but not a horizontal decrease of $[\mathrm{Fe} / \mathrm{H}]$. Here, the loop results from $\alpha-$ elements decreasing during the pause as Fe continues to be produced by Type Ia SNe, although less than in star formation regime. When star formation reignites the $[\alpha / \mathrm{Fe}]$ ratio increases and then decreases, as explained above.

The main differences between the two scenarios is the extra infall (starburst) and the efficiency of star formation. In the burst case, there is extra infall and the efficiency is high both before and during the starburst, whereas in the case of the pause in the star formation the efficiency is lower especially at the beginning but high after the reignition. The reason is that with a high star formation efficiency, as in some models of the classical bulge/bar (Matteucci et al. 2019), the gas is consumed quickly leaving too little to trigger star formation after the pause (a similar behavior was suggested by Matteucci et al. 2019 for the bulge). In the case of the bulge it was suggested that buckling of the bar could be the agent responsible for the pause in star formation.

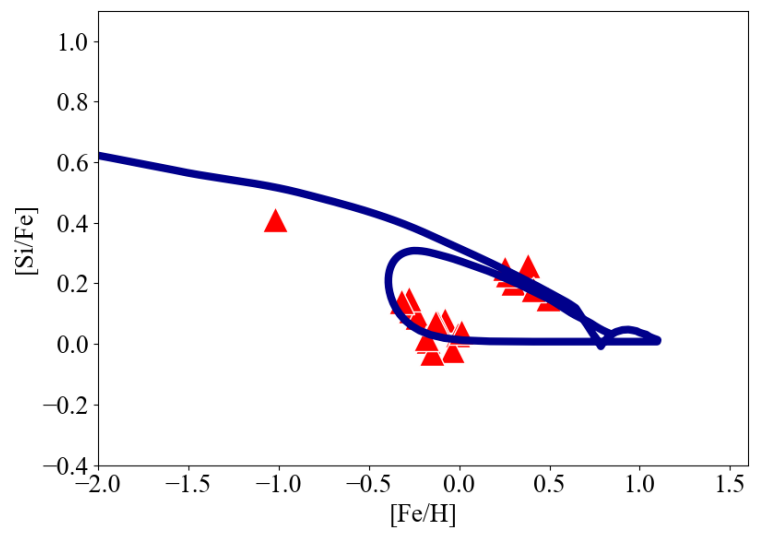

Figure 9. Chemical evolution model with a sudden accretion of primordial gas 2.5 Gyr ago adopting a Salpeter (1955) IMF. The blue line shows the $[\mathrm{Si} / \mathrm{Fe}]$ vs. $[\mathrm{Fe} / \mathrm{H}]$ trend of the gas from which stars are formed. Simplified, the line follows a time line from the upper left, down towards the bottom right as gas is consumed by star formation and enriched by Type II SNe and Type Ia SNe. A sudden infall of primordial or slightly enriched gas dilutes the gas resulting in a horizontal line towards the left. As the density of the gas increases the star formation incerases, for instance in a burst if the gas infall is rapid enough, at which an enrichment of Si by Type II SNe occurs until the time when the burst is diluted by the Type I SNe. During periods of low density few or no stars are formed, and therefore we do not expect observe stars uniformly populating the track.

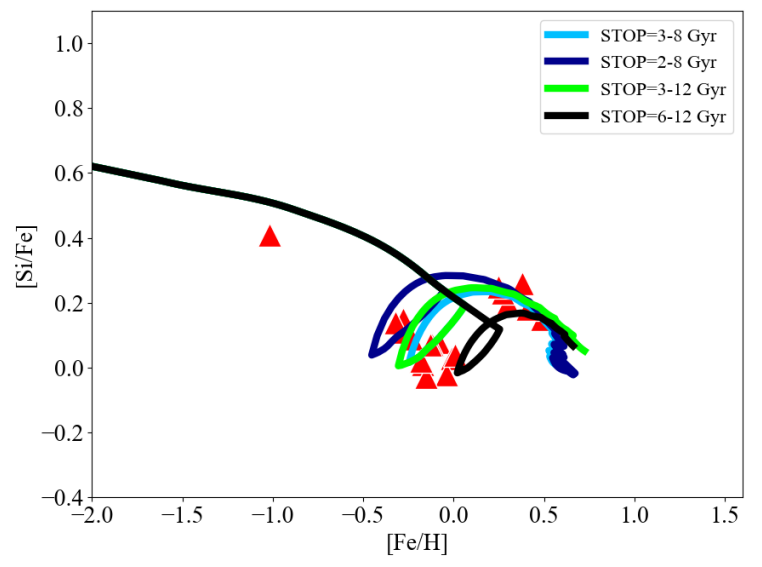

Figure 10. Chemical evolution models varying the temporal extent of the stop in star formation with a Salpeter (1955) IMF. All the cases are characterized by a re-ignition of the SF after the stop. See the description in Figure 9 for how to interpret the line.

Further investigations into modeling the alpha enrichment of super solar metallicity stars in the NSC and vicinity is called for, and we will investigate this in an upcoming paper.

\section{CONCLUSIONS}


We report the first trend of $[\mathrm{Si} / \mathrm{Fe}]$ vs. $[\mathrm{Fe} / \mathrm{H}]$ for giants in the Nuclear Star Cluster and vicinity. The new trend differs clearly from the local disk; giants with supersolar metallicity show a +0.2 dex enhancement in $[\mathrm{Si} / \mathrm{Fe}]$ relative to the stars in the local disk. Such an enhancement is also exceeding the values measured in the bulge.

In agreement with many studies of the inner and outer bulge, we find the most metal rich stars in the NSC to reach $[\mathrm{Fe} / \mathrm{H}]=+0.5$, and exhibit no evidence of more extreme composition.

We emphasize that our current sample size is modest, and it is now of critical importance to increase the sample size, and to seek confirmation of the trend using other alpha elements. This will allow us to perform a detailed analysis of the origin and evolution of the NSC populations by exploring different chemical enrichment scenarios.

\section{ACKNOWLEDGMENTS}

We thank Anish Amarsi for hosting B.T. in Heidelberg and assisting with the NLTE calculations. We also thank Mattia Sormani for his comments and suggestions. B.T. and N.R. acknowledges support from the Swedish Research Council, VR (project number 621-2014-5640), Funds from Kungl. Fysiografiska Sällskapet i Lund. (Stiftelsen Walter Gyllenbergs fond and Märta och Erik Holmbergs donation), and from the project grant "The New Milky" from the Knut and Alice Wallenberg foundation. M.S. acknowledges the Programme National de Cosmologie et Galaxies (PNCG) of CNRS/INSU, France, for financial support. R.M.R. acknowledges financial support from his late father Jay Baum Rich. F.R. acknowledges support from the Knut and Alice Wallenberg Foundation. A portion of this work was done at the Sexten Center for Astrophysics; the authors acknowledge their hospitality.

The data presented herein were obtained at the W. M. Keck Observatory, which is operated as a scientific partnership among the California Institute of Technology, the University of California and the National Aeronautics and Space Administration. The Observatory was made possible by the generous financial support of the W. M. Keck Foundation. The authors wish to recognize and acknowledge the very significant cultural role and reverence that the summit of Mauna Kea has always had within the indigenous Hawaiian community. We are most fortunate to have the opportunity to conduct observations from this mountain.

\section{Facilities: KECK:II (NIRSPEC)}

Software: SME (Valenti \& Piskunov 1996, 2012), REDSPEC (Kim et al. 2015), IRAF (Tody 1993), BSYN \& EQWIDTH (Gustafsson et al. 2008)

\section{REFERENCES}

Abolfathi, B., Aguado, D. S., Aguilar, G., et al. 2018, ApJS, 235, 42

Amarsi, A. M., \& Asplund, M. 2017, MNRAS, 464, 264

Antonini, F., Capuzzo-Dolcetta, R., Mastrobuono-Battisti, A., \& Merritt, D. 2012, ApJ, 750, 111

Arca-Sedda, M., Capuzzo-Dolcetta, R., Antonini, F., \& Seth, A. 2015, ApJ, 806, 220

Barber, R. J., Tennyson, J., Harris, G. J., \& Tolchenov, R. N. 2006, MNRAS, 368, 1087

Belyaev, A. K., Yakovleva, S. A., \& Barklem, P. S. 2014, A\&A, 572, A 103

Bensby, T., Yee, J. C., Feltzing, S., et al. 2013, A\&A, 549, A147

Blanton, M. R., Bershady, M. A., Abolfathi, B., et al. 2017, AJ, 154,28

Böker, T. 2010, in IAU Symposium, Vol. 266, Star Clusters: Basic Galactic Building Blocks Throughout Time and Space, ed. R. de Grijs \& J. R. D. Lépine, 58
Brooke, J. S. A., Bernath, P. F., Western, C. M., et al. 2016, JQSRT, 168, 142

Calura, F., \& Menci, N. 2009, MNRAS, 400, 1347

Capuzzo-Dolcetta, R. 1993, ApJ, 415, 616

Carr, J. S., Sellgren, K., \& Balachandran, S. C. 2000, ApJ, 530, 307

Cunha, K., Sellgren, K., Smith, V. V., et al. 2007, ApJ, 669, 1011

Davies, B., Origlia, L., Kudritzki, R.-P., et al. 2009, ApJ, 694, 46

Demarque, P., Woo, J.-H., Kim, Y.-C., \& Yi, S. K. 2004, ApJS, 155,667

Di Matteo, P. 2016, PASA, 33, e027

Do, T., Kerzendorf, W., Konopacky, Q., et al. 2018, ApJL, 855, L5

Do, T., Kerzendorf, W., Winsor, N., et al. 2015, ApJ, 809, 143

Dong, H., Schödel, R., Williams, B. F., et al. 2017, MNRAS, 471, 3617 
Eisenhauer, F., Abuter, R., Bickert, K., et al. 2003, in Instrument Design and Performance for Optical/Infrared Ground-based Telescopes, ed. M. Iye \& A. F. M. Moorwood, Vol. 4841, International Society for Optics and Photonics (SPIE), 1548

Emsellem, E., Renaud, F., Bournaud, F., et al. 2015, MNRAS, 446, 2468

Feldmeier-Krause, A., Kerzendorf, W., Neumayer, N., et al. 2017, MNRAS, 464, 194

Feldmeier-Krause, A., Neumayer, N., Schödel, R., et al. 2015, A\&A, 584, A2

Ferraro, F. R., Massari, D., Dalessandro, E., et al. 2016, ApJ, 828, 75

Fritz, T. K., Chatzopoulos, S., Gerhard, O., et al. 2016, ApJ, 821, 44

Gallego-Cano, E., Schödel, R., Nogueras-Lara, F., et al. 2020, arXiv e-prints, arXiv:2001.08182

Genzel, R., Eisenhauer, F., \& Gillessen, S. 2010, Reviews of Modern Physics, 82, 3121

Goorvitch, D. 1994, ApJSS, 95, 535

Grevesse, N., Asplund, M., \& Sauval, A. J. 2007, SSRv, 130, 105

Grieco, V., Matteucci, F., Ryde, N., Schultheis, M., \& Uttenthaler, S. 2015, MNRAS, 450, 2094

Guillard, N., Emsellem, E., \& Renaud, F. 2016, MNRAS, 461, 3620

Gustafsson, B., Edvardsson, B., Eriksson, K., et al. 2008, A\&A, 486, 951

Johnson, C. I., Rich, R. M., Kobayashi, C., Kunder, A., \& Koch, A. 2014, THE ASTRONOMICAL JOURNAL, 148, 67

Johnson, J. W., \& Weinberg, D. H. 2019, arXiv e-prints, arXiv: 1911.02598

Kim, S., Prato, L., \& McLean, I. 2015, REDSPEC: NIRSPEC data reduction, Astrophysics Source Code Library, ascl:1507.017

Kovtyukh, V. V., Andrievsky, S. M., Martin, R. P., et al. 2019, MNRAS, 489, 2254

Krumholz, M. R., \& Kruijssen, J. M. D. 2015, MNRAS, 453, 739

Langhoff, S. R., \& Bauschlicher, C. W. 1993, Chem. Phys. Letters, 211,305

Launhardt, R., Zylka, R., \& Mezger, P. G. 2002, A\&A, 384, 112

Livingston, W., \& Wallace, L. 1991, An atlas of the solar spectrum in the infrared from 1850 to $9000 \mathrm{~cm}-1$ (1.1 to 5.4 micrometer) (NSO Technical Report, Tucson: National Solar Observatory, National Optical Astronomy Observatory, 1991)

Loose, H. H., Kruegel, E., \& Tutukov, A. 1982, A\&A, 105, 342

Majewski, S. R., Schiavon, R. P., Frinchaboy, P. M., et al. 2017, AJ, 154, 94

Mapelli, M., Hayfield, T., Mayer, L., \& Wadsley, J. 2012, ApJ, 749,168

Mastrobuono-Battisti, A., Perets, H. B., Gualand ris, A., Neumayer, N., \& Sippel, A. C. 2019, MNRAS, 490, 5820

Matsunaga, N., Fukue, K., Yamamoto, R., et al. 2015, ApJ, 799, 46
Matteucci, F. 2012, Chemical Evolution of Galaxies (Springer Berlin Heidelberg)

Matteucci, F., Grisoni, V., Spitoni, E., et al. 2019, MNRAS, 487, 5363

McLean, I. S. 2005, in High Resolution Infrared Spectroscopy in Astronomy, ed. H. U. Käufl, R. Siebenmorgen, \& A. F. M. Moorwood (Springer-Verlag Berlin Heidelberg), 25

McLean, I. S., Prato, L., McGovern, M. R., et al. 2007, ApJ, 658, 1217

Milosavljević, M. 2004, ApJL, 605, L13

Minniti, D., Contreras Ramos, R., Zoccali, M., et al. 2016, ApJL, 830, L14

Morris, M., \& Serabyn, E. 1996, ARA\&A, 34, 645

Najarro, F., Figer, D. F., Hillier, D. J., Geballe, T. R., \& Kudritzki, R. P. 2009, ApJ, 691, 1816

Nandakumar, G., Ryde, N., Schultheis, M., et al. 2018, MNRAS, 478,4374

Neumayer, N. 2017, in IAU Symposium, Vol. 316, Formation, Evolution, and Survival of Massive Star Clusters, ed. C. Charbonnel \& A. Nota, 84

Neumayer, N., Seth, A., \& Boeker, T. 2020, arXiv e-prints, arXiv:2001.03626

Nogueras-Lara, F., Schödel, R., Dong, H., et al. 2018, A\&A, 620, A83

Nogueras-Lara, F., Schödel, R., Gallego-Calvente, A. T., et al. 2019a, Nature Astronomy, 4

-. 2019b, A\&A, 631, A20

Origlia, L., Massari, D., Rich, R. M., et al. 2013, ApJL, 779, L5

Origlia, L., Rich, R. M., Ferraro, F. R., et al. 2011, ApJL, 726, L20

Origlia, L., Mucciarelli, A., Fiorentino, G., et al. 2019, ApJ, 871, 114

Pehlivan, A., Nilsson, H., \& Hartman, H. 2015, A\&A, 582, A98

Pehlivan Rhodin, A., Hartman, H., Nilsson, H., \& Jönsson, P. 2017, A\&A, 598, A102

Pfuhl, O., Fritz, T. K., Zilka, M., et al. 2011, ApJ, 741, 108

Ramírez, S. V., Sellgren, K., Carr, J. S., et al. 2000, ApJ, 537, 205

Renaud, F., Bournaud, F., Emsellem, E., et al. 2013, MNRAS, 436, 1836

Rich, R. M., Ryde, N., Thorsbro, B., et al. 2017, AJ, 154, 239

Ryde, N., Fritz, T. K., Rich, R. M., et al. 2016a, ApJ, 831, 40

Ryde, N., \& Schultheis, M. 2015, A\&A, 573, A14

Ryde, N., Schultheis, M., Grieco, V., et al. 2016b, AJ, 151, 1

Salpeter, E. E. 1955, ApJ, 121, 161

Schödel, R., Feldmeier, A., Kunneriath, D., et al. 2014, A\&A, 566, A47

Schultheis, M., Ryde, N., \& Nandakumar, G. 2016, A\&A, 590, A6

Scott, P., Asplund, M., Grevesse, N., Bergemann, M., \& Sauval, A. J. 2015a, A\&A, 573, A26

Scott, P., Grevesse, N., Asplund, M., et al. 2015b, A\&A, 573, A25 
Seth, A. C., Neumayer, N., \& Boeker, T. 2019, arXiv e-prints, arXiv:1908.00022

Smith, V. V., Cunha, K., Shetrone, M. D., et al. 2013, ApJ, 765, 16

Sneden, C., Cowan, J. J., Kobayashi, C., et al. 2016, ApJ, 817, 53

Spitoni, E., Silva Aguirre, V., Matteucci, F., Calura, F., \& Grisoni, V. 2019, A\&A, 623, A60

Støstad, M., Do, T., Murray, N., et al. 2015, ApJ, 808, 106

Thorsbro, B., Ryde, N., Rich, R. M., et al. 2017, Proceedings of the International Astronomical Union, 13, 372-373

Thorsbro, B., Ryde, N., Schultheis, M., et al. 2018, ApJ, 866, 52
Tody, D. 1993, in ASP Conf. Ser. 52: Astronomical Data Analysis Software and Systems II, ed. R. J. Hanisch, R. J. V. Brissenden, \& J. Barnes, 173

Tremaine, S. D., Ostriker, J. P., \& Spitzer, Jr., L. 1975, ApJ, 196, 407

Valenti, J. A., \& Piskunov, N. 1996, A\&AS, 118, 595

—. 2012, SME: Spectroscopy Made Easy, ascl:1202.013, astrophysics Source Code Library 